Culture et histoire dans l'espace roman

$6 \mid 2011$

Figures du pouvoir dans la littérature hispanoaméricaine

\title{
Les figures invisibles d'un pouvoir omniprésent
}

Relations sociales et dictature dans le roman argentin El Colectivo de Eugenia Almeida

\section{Margaux Revol}

\section{CpenEdition}

\section{Journals}

Édition électronique

URL : https://journals.openedition.org/cher/9802

DOI : $10.4000 /$ cher.9802

ISSN : 2803-5992

Éditeur

Presses universitaires de Strasbourg

Édition imprimée

Date de publication : 30 juin 2011

Pagination : $91-100$

ISBN : 978-2-35410-033-9

ISSN : 1968-035X

Référence électronique

Margaux Revol, « Les figures invisibles d'un pouvoir omniprésent », reCHERches [En ligne], 6 | 2011, mis en ligne le 17 décembre 2021, consulté le 31 janvier 2022. URL : http://journals.openedition.org/cher/ 9802 ; DOI : https://doi.org/10.4000/cher.9802

\section{(c) (i) (2)(2)}

Ce(tte) œuvre est mise à disposition selon les termes de la Licence Creative Commons Attribution -

Pas d'Utilisation Commerciale - Partage dans les Mêmes Conditions 4.0 International. 


\title{
Les figures invisibles d'un pouvoir omniprésent : relations sociales et dictature dans le roman argentin El Colectivo de Eugenia Almeida
}

\author{
Margaux Revol \\ IHEAL, Paris III - Sorbonne Nouvelle
}

\begin{abstract}
$\mathrm{N}^{\mathrm{e} e}$ ée en 1972 à Córdoba, Eugenia Almeida n'était qu'une enfant lors de la dictature militaire argentine de 1976-1983. Pourtant, elle se souvient d'un climat, lourd de silence et de faux-semblants ${ }^{1}$. Dans son premier roman publié d'abord en 2005, El Colectivo, elle retranscrit cette atmosphère étouffante de la période du Proceso de Reorganización nacional, sans toutefois faire appel à la création manichéenne de héros et d'anti-héros, qui résistent ou au contraire soutiennent activement le régime militaire. Le pouvoir dictatorial semble n'être dans le roman qu'une rumeur lointaine, alors même qu'il est bien palpable. En 1977, dans ce hameau reculé d'Argentine qu'Eugenia Almeida imagine comme cadre, le quotidien des villageois est perturbé par la traque de deux «subversifs» qui paralyse les transports locaux. Ce village anonyme, simple tache sur la carte de l'Argentine, est pour l'auteure le laboratoire fictionnel d'une société en réduction. Ce choix qu'elle opère va lui permettre d'isoler les réactions individuelles face à la répression, et de mettre en relief des traits psychologiques collectifs. La typologie qui s'en dégage fait une large part aux éléments réactionnaires, porte-voix inquiétants de la dictature militaire. Les manifestations du pouvoir dans le roman ne seront donc pas tant des incarnations physiques de la dictature militaire, mais bien plutôt des signes, dans les discours mêmes des villageois,

1 «Los niños son expertos en detectar climas. Creo que algo de esa experiencia quedó dentro mío». Interview du journal Los Andes, 30 mai 2009, en ligne: http://www. losandes.com.ar/notas/2009/5/30/cultura-426609.asp
\end{abstract}


relayant et symbolisant très souvent les conceptions et valeurs autoritaires du gouvernement de facto.

\section{Une présence diffuse et immatérielle du pouvoir dictatorial}

El Colectivo pourrait être résumé comme l'histoire d'un encerclement: l'autobus reliant quotidiennement le village aux grandes villes argentines passe sans s'arrêter, et la voie ferrée est bloquée sur ordre de l'armée, qui cherche à abattre des subversifs signalés dans la zone. Pourtant, cette réalité de la dictature, la véritable cartographie de la terreur divisant le pays en zones d'opérations répressives orchestrées par les militaires, n'apparait pas aussi clairement dans le roman. Il y est seulement question de cet autobus qui ne veut plus s'arrêter, et de ces deux étrangers de passage dans le village et partis à la hâte, perturbations du quotidien des villageois, tout autant qu'objets de curiosité.

En fait, le pouvoir entendu comme autorité émanant du gouvernement de facto et exercée par les militaires, est physiquement à peine présent dans le roman, à peine visible, et à peine évoqué par les personnages. Exception faite d'un bref passage à la toute fin du récit, point de soldat dans le village, ni d'autre uniforme que celui - devenu trop étroit - d'un commissaire débonnaire. La référence même à cette dictature n'est faite qu'à travers l'expression floue de "esto del gobierno militar», lâchée par le commissaire lui-même (Almeida 2007: 83), et la date de 1977 n'est connue qu'au détour d'une conversation banale entre deux villageois. C'est donc à travers d'autres éléments que le lecteur peut identifier la menace répressive du pouvoir autoritaire. À travers une ambiance, d'abord, le récit s'ouvrant sur un ciel menaçant, une atmosphère étouffante que la nervosité des chiens qui aboient vient exacerber. Ce climat est symboliquement déjà porteur de mauvais présages, tout comme les inquiétudes précoces de quelques personnages lucides et humains - l'hôtelier Rubén et le messager Gómez - qui ont très vite l'intuition qu'il se passe quelque chose de grave. Cette inquiétude est entretenue par les ondes de la radio qui informe puis désinforme capricieusement sur la situation: après avoir annoncé que deux individus étaient recherchés dans la zone, la radio fait brusquement silence sur l'état des recherches. C'est par ce silence radio que les villageois comprennent la disparition de ces individus, que l'armée a assassinés. De tels procédés assoient et alimentent le pouvoir pervers de la dictature: on fait oublier l'événement en n'y faisant plus référence, mais en ne démentant pas l'horreur, on laisse planer la menace de la répression. 
Plus fortement encore, l'autorité du gouvernement militaire transparait dans celle des représentants traditionnels du pouvoir local: l'avocat Ponce, et le commissaire. Car chacun d'eux va voir les limites de son autorité déplacées, voire annulées le temps de la traque. L'existence et la toutepuissance dictatoriales deviennent alors palpables, en creux, dans la remise en question de l'autorité de ces personnages qui perdent, chacun à leur manière, de leur ascendant sur les autres membres de la société villageoise. Dire qu'ils perdent de l'influence et des prérogatives, c'est dire que quelqu'un d'autre se les appropie. Le tout-puissant gouvernement militaire, faisant $\mathrm{fi}$ des hiérarchies civiles traditionnelles, phagocyte tous les représentants de l'ordre. Ainsi le commissaire ne sait plus à quelle hiérarchie se vouer ${ }^{2}$, et ses interactions ponctuelles avec les militaires - des conversations téléphoniques régulières - sont souvent mises en scène sous forme de dialogue au discours direct où l'impératif est omniprésent. Cette puissance militaire qui le dépasse est rendue plus menaçante encore dans la conversation téléphonique qui retranscrit exclusivement les paroles du commissaire: "-Sí, señor; soy yo. No, señor. Sí, señor; estuvo acá. Salió para Pozo del Sauce. Anteanoche. No, no llegó. Sí, señor. Con una mujer. No, no lo sé. Sí, señor. Hasta luego » (95). Ici, le lecteur ignore jusqu'à l'identité de l'interlocuteur, et le silence autour de cet autre versant de la conversation, dissimulée au lecteur, est une façon habile de la part de l'auteure de faire croître l'inquiétude. Si l'on en croit Miguel Ángel Asturias, en effet, à propos de El Señor presidente, celui-ci est d'autant plus dangereux et paraît d'autant plus puissant qu'on ne le voit jamais ${ }^{3}$. Les ordres émanant de cette autorité supérieure et écrasante sont toujours issus dans le roman d'une troisième personne du pluriel de tournure impersonnelle ${ }^{4}$, qui vient renforcer l'invisibilité, l'impalpabilité de ce pouvoir dont personne ne désigne ses décideurs, ses détenteurs.

Quant à Ponce, l'avocat, il ne se remet pas de l'humiliation essuyée lorsque le bus est passé devant lui sans s'arrêter, et ce plusieurs jours de suite alors

2 «Eso también es un lío. De golpe, ahora tengo tantos superiores que no sé bien cómo es el orden de mando. Y con esto del gobierno militar..., no sé, no sé si responder a mi mando superior o a un militar con rango menor...» (83).

3 «[...] invisible como Dios, pues entre menos corporal aparezca, más mitológico se le considera. La fascinación que ejerce en todos, aun en sus enemigos, el halo de ser sobrenatural que lo rodea, todo concurre a la reactualización de lo fabuloso, fuera de un tiempo cronológico». Conférence de M.A. Asturias dictée à Turin, intitulée «El Señor Presidente como mito", in Giuseppe Bellini, 1966, La narrativa di Miguel Ángel Asturias, Instituto Editoriale Cisalpino, Milán, p.36.

4 «Me mandaron una orden. No desde Córdoba, directamente de Buenos Aires. Me dijeron que dejara la barrera baja» (84); «A mí me mandan una orden y yo la hago cumplir» (82). 
même que les autres villageois étaient témoins d'une telle avanie. Ce qu'il interprète comme un affront personnel, dans des discours où foisonnent les occurrences du pronom personnel de première personne, est en réalité le fruit d'une décision qui le dépasse: l'ordre intimé par les militaires aux chauffeurs de bus de ne pas s'arrêter dans les villages, afin de mieux contrôler les voyageurs dans la région. Son égocentrisme l'empêche de voir que des forces supérieures sont à l'œuvre, quand cela n'échappe pas au contraire à l'hôtelier lucide Rubén, qui finit par lui déclarer familièrement: «Ponce, usted no es el centro del mundo» (78), phrase d'autant plus violente que son auteur se dispense délibérement du titre de «maître» pour s'adresser à l'avocat. Dès le début, en effet, Ponce semble devenu transparent: le chauffeur a l'air de ne pas l'avoir vu, puisqu'il ne s'est pas arrêté, et Gómez ne l'entend pas lorsqu'ils se croisent. Plus que de la perte de son ascendant social, Ponce souffre de l'indifférence à laquelle il est ravalé: «Todos lo vieron hacer el ridículo. Dar una orden y ni siquiera obtener desobediencia. Lo que habían hecho era ignorarlo. Hacer de cuenta que él era uno más", pense-t-il sous forme de discours indirect libre (121).

Ainsi, si l'on peut deviner le pouvoir dictatorial à travers des jeux de non-dits et d'ombres portées, le roman comporte somme toute très peu d'indices concrets qui prouveraient que les villageois sont conscients de l'existence de la dictature. Cela explique d'ailleurs que Victoria, la sœur de l'avocat Ponce, décide de quitter la ville où elle habite ordinairement pour se retirer provisoirement dans ce village: elle qui est confrontée à la réalité de la répression, aux échanges de tirs et aux cadavres, elle espère que le village n'aura pas été atteint par l'ambiance mortifère de la dictature: «Quizás allá los rumores, los chirridos, los zarpazos, los golpes, las corridas, los moretones, las botas, los disparos no hayan llegado. No todavía» (108). Mais c'est ce «no todavía», ce "pas encore» de mauvais augure qui vient sonner le glas de la tranquilité et de la feinte ignorance.

À première vue en effet, c'est un village sans histoire, étranger au pouvoir autoritaire. Anonyme et perdu dans l'immensité argentine, il semble au début du roman ne pas avoir été atteint par l'onde de choc de la dictature. Lorsqu'un autochtone sort une coupure de journal, en général ce n'est pas pour en consulter les informations mais pour en noircir la grille des mots croisés. Eugenia Almeida développe plusieurs stratégies d'écriture pour figurer le poids des habitudes villageoises, telles qu'une esthétique narrative et lexicale de la répétition - les différents personnages posent les mêmes questions, répandent les mêmes rumeurs, emploient les mêmes mots - ou la présentation in medias res des personnages- tous désignés par leurs 
prénoms comme s'ils étaient bien connus du lecteur, ce qui vient mimer cette familiarité propre aux petits villages. Bref, comme le signale l'un des personnages: «Acá las cosas tienden a repetirse» (78).

Mais derrière ces apparences, les discours des personnages et les rapports entre eux en disent long sur le potentiel autoritaire de chacun. Derrière de banales discussions de comptoir, émergent des conceptions autoritaires qui se font le relais du discours dictatorial.

\section{L'ombre du pouvoir : la société comme réceptacle et relais de l'ordre dictatorial}

Si aucun personnage n'est un héros ni un anti-héros, si personne au cours du roman n'intervient activement pour empêcher ou au contraire favoriser l'assassinat des deux personnes traquées par l'armée, ce sont les attitudes et discours de ces villageois qui viennent figurer le pouvoir autoritaire dans ses traits historiques caractéristiques. C'est donc bien l'autoritarisme latent chez certains personnages du village qui nous intéresse ici, selon l'acception qu'en fait Theodor Adorno dans ses études sur la personnalité autoritaire:

Ce que disent les gens et, à un moindre degré, ce qu'ils pensent réellement dépend très largement du climat d'opinions dans lequel ils vivent; mais lorsque ce climat change, certains individus s'adaptent eux-mêmes beaucoup plus vite que d'autres. [...] En d'autres termes, les individus diffèrent dans leur réceptivité à la propagande démocratique, dans leur disponibilité à exhiber des tendances anti-démocratiques (2007: 11-12).

Il s'agit pour nous de montrer l'envers du décor, c'est-à-dire à quel point le pouvoir, en tant que volonté normative et autorité fantasmée, est omniprésent dans les discours et schémas mentaux des villageois de $E l$ Colectivo.

Car derrière la routine, figurée par l'esthétique de la répétition, il y a une véritable résistance au changement: on peut en trouver un exemple significatif dans le fait que la coiffeuse Rita ou le pharmacien ne croient pas une seule seconde que Primitivo, le garde-barrière, a arrêté de boire. Ce dernier était certainement un alcoolique notoire quelques années auparavant, mais ce n'est plus le cas. Le fait de plaisanter encore sur le sujet vient confirmer ce qu'explique Rubén à Gómez: «En este lugar no se toleran los cambios. Ni siquiera los positivos» (72). Cette résistance aux changements est symptomatique d'opinions réactionnaires.

"Viste cómo son las cosas, todo se va sabiendo", déclare Gómez à Primitivo (86). Tout se sait dans le village. Or derrière l'extrême familiarité 
ambiante se profilent la tendance inquiétante à la surveillance de tout le monde par tous, selon un schéma général narrativement proche du panoptique (Foucault 1975), puisque dans ce microcosme qu'est le village, les individus savent tout de tous, et tentent de s'imposer des normes de conduite rigides en faisant peser la menace d'une punition. Or ne nous y trompons pas, c'est bien à la forme la plus essentielle de "pouvoir» que nous avons à faire ici: les relations de pouvoir «s'exercent pour une part extrêmement importante à travers la production et l'échange de signes» (Foucault 1982: 234). Ces signes sont des valeurs normatives que les personnes tentent de s'imposer les unes aux autres, ici des valeurs hygiénistes, réactionnaires et conservatrices, à l'image des convictions de la junte militaire.

La narration omnisciente met le lecteur lui-même dans la position indiscrète de celui qui peut connaître tous les faits et gestes des personnages, puisqu'elle lui donne des yeux et des oreilles partout. La large part faite au style indirect libre, livrant au lecteur les pensées les plus intimes ou inavouables des personnages, fait de lui un surveillant des autres, selon une radiographie narrative des esprits. La familiarité devient alors un obstacle à l'intimité de chacun, puisqu'elle repousse toujours plus loin les frontières de la sphère privée. Ainsi peut s'épanouir l' "agressivité autoritaire», définie par Adorno à l'échelle individuelle comme la «tendance à espionner et à condamner, refuser et punir les personnes qui violent les valeurs conventionnelles» (Adorno 2007), caractéristique du gouvernement de facto lui-même par le biais de ses redoutables services d'information. Les dialogues favorisent en effet, comme dans la réalité, la propagation de rumeurs bien souvent infondées. La jeune fille Fuentes par exemple, évoquée à plusieurs reprises, est étiquetée comme une prostituée dans tout le village pour avoir été vue trop souvent auprès des ouvriers, alors qu'elle devait probablement appartenir à des groupes de résistance au régime. La dénonciation et l'arrestation de cette jeune fille sont présentées par Marta, épouse écervelée de Ponce, comme le triomphe de la justice sur la «mauvaise vie». Dans l'esprit de Marta, délit et péché sont équivalents, écho très clair de la réalité de cette dictature militaire qui fait de l'Église catholique le garant moral du bienfondé du régime. Marta aggrave son agressivité autoritaire d'une véritable obsession pour le sexe, autre caractéristique classique de la personnalité autoritaire. En effet, Marta se montre impitoyable dans ses discours envers les personnes adultères ou libérées sexuellement, qu'elle voudrait voir condamnées, alors qu'elle-même est tombée enceinte, trente ans plus tôt, en s'offrant à un inconnu dans une ruelle sombre et tous jupons levés. 
La prétendue moralité de Marta, de Ponce ou de Rita notamment, révèle en fait une conception exclusivement punitive de la vie et de la norme juridique. Marta en fait la preuve lorsqu'elle se réjouit avec violence et vulgarité de la punition de la jeune Fuentes qu'elle traite littéralement de «pute». L'histoire même de l'arrivée de Ponce et Marta dans le village est déjà l'histoire d'une punition originelle, aux sens religieux et moral du terme: Ponce, humilié d'avoir eu à épouser Marta pour cacher sa grossesse alors qu'elle a finalement perdu l'enfant, décide d'envoyer Marta dans l'enfer du village perdu, et se condamne lui-même à cet isolement pour mieux la faire souffrir. Bref, le recours (en acte ou potentiel) à la répression pour régler les conflits est non seulement un parallèle immédiat à dresser avec les méthodes de la junte militaire, mais aussi, si l'on en croit la sociologue María Virginia Ameztoy, une mise en abyme de l'histoire argentine dans son ensemble:

Asimismo, aparece como una constante el hecho de dirimir los conflictos sociales y políticos por medio de la represión. La suma de estos factores ha conformado una estructura social heterogénea y que en determinadas etapas de nuestra historia - especialmente durante la implantación de regímenes militares dictatoriales - acusó un alto grado de desintegración social, resquebrajadora de los lazos sociales y tendiente a la generación de pautas conductales de tipo autoritario por parte de los actores sociales (Ameztoy 1996: 12).

Ce qui frappe aussi dans les discours de Marta et de Rita, c'est la stéréotypie avec laquelle elles se figurent les personnes dites «subversives». La notion de «subversion» pour elles ne semble pas avoir de sens politique, elle se confond plutôt avec celle de déviance morale, puisqu'elles ravalent au même rang toutes les femmes qui leur semblent avoir des mœurs légères. Tout au long du roman, elles désignent les «subversifs" par le pronom à la fois flou et péjoratif «esos», qui prouve non seulement une volontaire mise à distance vis-à-vis des personnes mentionnées mais également une profonde méconnaissance des réalités politiques. Poussée à l'extrême, cette tendance à penser les autres selon des schémas rigides devient "stéréopathie»: on finit par classer les gens et les objets dans des cases fantasmées qui ne correspondent plus à la réalité (Adorno 2007 : 241). Car l'autre versant de la stéréotypie, c'est la tendance à opérer des dichotomies rigides entre le bien et le mal, nous et les autres, les bons et les mauvais etc. A cet égard, les travaux de la sociologue Mariana Caviglia, sur les classes moyennes argentines durant la dictature militaire de 1976-1983, nous permettent de comprendre comment ont réagi les personnes ordinaires (n'ayant ni vraiment soutenu 
la junte, ni vraiment fait acte de résistance) face à la répression. Selon elle, cela a été tout à fait répandu et symptomatique qu'une large majorité des gens ait décidé, pour se protéger, de maintenir une distance forte avec tout ce qui pouvait les faire confondre avec les subversifs. Dès lors, les subversifs deviennent l'Autre radical, celui que l'on ne veut pas approcher, que l'on rejette au dehors. Le manichéisme est omniprésent dans les discours de Marta et de Rita. Cela est manifeste lorsque Rita parle des subversifs dans l'altérité d'une troisième personne du pluriel, alors même qu'elle désigne les soldats par le pronom personnel «nosotros» dans lequel elle s'inclut («los nuestros» par opposition aux «guerrilleros»). Elle va jusqu’à nier la possibilité qu’il existe des subversifs dans son environnement: « ¿Cómo van a ser de acá? No. Acá no tenemos. Gracias a dios. Esos aparecen en la ciudad. Andan por ahí, metiéndose en cosas raras...Usted sabe...»(133). Il s'agit bien, comme l'explique Mariana Caviglia, de ne pas franchir l'espace réservé aux Autres: «no transitar los espacios de los Otros» (Caviglia 2006: 180).

Le manichéisme excluant est largement présent dans les relations entre les villageois, dans la mesure où le village est géographiquement et socialement scindé en deux parties, dont la frontière est matérialisée par la voie ferrée. Il y a "este lado" et "el otro lado», dont les dénominations ne relèvent pas de la situation relative dans l'espace, mais sont établies et voulues fixes par les acteurs sociaux dominants qui habitent tous le même côté du village: le médecin, le pharmacien, la coiffeuse etc.

El pueblo real, el pueblo verdadero, está de este lado de las vías. Y usted lo sabe. Del otro lado hay unas chacras, el campo, un par de baqueanos, los ranchos [...] putas. Putas, delincuentes, choros, vagos, borrachos. No son como nosotros (29).

La violence du vocabulaire montre l'acharnement des vainqueurs réactionnaires, qui, même sous la dictature militaire garantissant les valeurs qu'ils chérissent, ne pacifient pas leur discours.

Ce côté-ci contre ce côté-là, le village contre la ville (siège de tous les vices), les subversifs contre «nous» les gens «bien», les prostituées contre les femmes «comme il faut»... Toute cette énergie investie dans l'obstination à opposer catégoriquement des réalités vécues comme radicalement bonnes ou mauvaises est bien la preuve que cette société partage la vision intolérante et manichéenne du gouvernement militaire, et qu'elle se fait ainsi relais inconscient de la junte, puisqu'elle n'a pas besoin de la présence de soldats pour répercuter un ensemble de normes autoritaires. Elle est aussi le reflet 
d'une dichotomie historique de la société argentine, comme l'explicite María Virginia Ameztoy:

En nuestra historia y en la génesis de sus luchas, los efectos de dicha estructuración social binaria aparecen como una constante: unitarios y federales, criollos e indígenas, Buenos Aires y la Confederación, civilización y barbarie, conservadores y liberales, criadores e invernadores, peronismo y anti-peronismo, militares y civiles... orden y subversión (Ameztoy 1996: 12).

En dernier lieu, le lecteur découvre avec quelle fascination morbide Rita ou Marta encensent les figures du pouvoir dictatorial, incarnées dans les soldats. Ce dernier trait caractéristique pour Theodor Adorno de la personnalité autoritaire, qu'il désigne comme le "complexe du pouvoir», consiste en l'admiration de ceux qui détiennent le pouvoir. Il faut lire ces lignes où Marta se pâme en évoquant sa rencontre avec des militaires:

Vinieron cuatro oficiales y se la llevaron. Vieras qué bien los policías, qué elegantes. No usaban uniformes, venían de traje, en un auto grande. [...] ¡Y vieras qué pinta! Los trajes que tenían los oficiales, el pelo bien cortito, el bigote perfecto. Vieras qué bien. [...] Limpian el pueblo, nos protegen (110-112).

Elles sont la preuve ultime de l'adhésion au régime, alors que, par ailleurs, le roman évoque à peine les figures proprement dites de ce pouvoir dictatorial.

La notion de «relais» plusieurs fois employée ici peut s'entendre dans son sens premier réservé à la vénerie: des chiens lancés derrière le gibier lorsque l'on part en chasse. La collectivité villageoise du Colectivo évoque, à travers les discours de ses représentants qui rallient les convictions de la junte militaire, une meute sauvage potentiellement dangereuse, aux opinions rances et intolérantes. Revenons au titre du roman: El colectivo, en espagnol d'Argentine, signifie bien sûr l'autobus, mais lo colectivo fait référence à la notion de collectif. Ce titre pose d'emblée la question de la cohésion, de la valeur du groupe. Car si les gens du village fonctionnent bien souvent comme un groupe ou sont référés par l'intermédiaire d'une troisième personne du pluriel impersonnelle qui les unit comme une entité collective, notamment lorsqu'ils se réunissent autour du bus qui ne passe pas, leur regroupement éphémère cache à peine l'individualisme et l'égoïsme de chacun:

Se oye el ruido del colectivo que acelera. Se oyen gritos, ayes, epa, ole. La gente festeja, se asombra, se divierte. Parece una turba transparente celebrando una corrida de toros. Sólo que nadie se pregunta quién es el torero y quién va a morir para el placer de los otros. Cuando el grupo se deshace y cada uno vuelve a lo suyo, cada quien por su camino, a sus petates, a sus pequeños disturbios... (87). 
Finalement, la question que pose l'auteure dans son roman est, de son propre aveu, celle qui anime également les questionnements de son second roman, La pièce du fond (Almeida 2010) à savoir ce que les gens sont prêts à faire pour les autres.

Dans El Colectivo, ce sont seulement trois personnages qui viennent dynamiter les faux-semblants de cette prétendue ignorance à propos de la répression dictatoriale. Rubén, Gómez, et Victoria brisent la loi du silence et condamnent par leur questionnement et leur esprit critique l'hypocrisie de tous ceux qui disent «je ne savais pas». Car c'est ce silence lourd d'omissions que ne tolère pas Eugenia Almeida, comme elle le confie lors d'une interview accordée à Página 12/:

Uno podía no digerirlo, pero yo tenía cinco años y me daba cuenta de que algo monstruoso estaba pasando. Por eso desconfío mucho de esa explicación; me parece mucho más plausible decir "yo sabía lo que pasaba y estaba muerto de miedo". Eso es totalmente comprensible 5 .

Avec El Colectivo, Almeida brosse un tableau saisissant et inquiétant de cette société argentine déchirée, disloquée, celle du tristement nommé Proceso de Reorganización Nacional, qui a réorganisé la société pour mieux en désintégrer tous les liens sociaux.

\section{Bibliographie}

Adorno, Theodor, 2007, Études sur la personnalité autoritaire, Paris, Allia. Almeida, Eugenia, 2007, El colectivo, Madrid, Roca.

Almeida, Eugenia, 2010, La pièce du fond, Paris, Métailié.

Ameztoy, María Virginia, 1996, "Autoritarismo, sociedad y estado en Argentina», in Violencia social y derechos humanos, Inés Aguirre (ss coord), Buenos Aires, Eudeba, en ligne: http://www.iigg.fsoc.uba.ar/conflictosocial/libros/violencia/ index.htm

Caviglia, Mariana, 2006, Dictadura, vida cotidiana y clases medias: una sociedad fracturada, Buenos Aires, Prometeo Libros.

Foucault, Michel, 1975, Surveiller et punir: naissance de la prison, Paris, Gallimard.

Foucault, Michel, 1994, «Le sujet et le pouvoir», in Dits et écrits, Paris, Gallimard, vol. IV, p. 222-243.

5 Interview accordée à Página 12/, 10 avril 2009, en ligne: http://www.pagina12.com.ar/ diario/suplementos/espectaculos/4-13472-2009-04-10.html 